\title{
Mitra esmamainimine Hetiidi kuninga Šuppiluliuma I ja Mitanni kuninga Sattiwaza lepingus?
}

\author{
Lühiuurimus indoiraani usundiloost*
}

\author{
Jaan Lahe \\ Eesti Evangeelse Luterliku Kiriku Usuteaduse Instituudi \\ piibliteaduste professor, \\ Tallinna Ülikooli Humanitaarteaduste instituudi \\ kultuuri- ja religiooniuuringute dotsent \\ usundiuurija@gmail.com \\ Vladimir Sazonov \\ Sisekaitseakadeemia sisejulgeoleku instituudi teadur, \\ Tartu Ülikooli humanitaarteaduste ja kunstide valdkonna \\ kultuuriteaduste instituudi Vana-Lähis-Ida uuringute vanemteadur \\ vladimir.sazonov@ut.ee
}

Teesid: 1907. aastal hetiidi kuninglikust arhiivist Hattuša linnas avastatud leping, mis on sõlmitud Hetiidi kuninga Šuppiluliuma I ja Mitanni kuninga Šattiwaza vahel 14. sajandil eKr, on äratanud uurijate tähelepanu sellega, et see sisaldab erinevate (hetiidi, assüüria, babüloonia jm) jumalanimede hulgas mitmeid nimesid, mille indoeuroopa päritolu on väljaspool kahtlust. Nimede esinemist lepingutekstis saab seletada Mitanni valitseva kihi indoeuroopa päritoluga. Indoeuroopa jumalanimede hulgas esineb ka $M i$-it-ra-aš-ši-il, mida on tõlgendatud jumal Mitra esmamainimisena.

Käesoleva artikli autorid juhivad eriliselt tähelepanu nime mitmuslikule vormile ja väidavad, et tõenäoliselt ei ole siin tegemist pärisnimega, vaid teatud jumalaterühma tähistusega, mida võiks tõlkida "lepingu(te)jumalad". Kuigi uurijad on olnud nime mitmusevormist teadlikud ka varem, on enamik jumal Mitrast kirjutanud teadlasi tõlgendanud nime ainsuslikuna ega ole autorite meelest pakkunud omalt poolt välja ka ühtegi veenvat nime tõlkevarianti.

Märksõnad: Hetiidid, indoeurooplased, leping, lepingujumalused, Mitanni riik, Mitra, Šattiwaza, Šuppiluliuma I 


\section{Sissejuhatus}

1907. a. avastati hetiidi kuninglikust arhiivist Hattušast (praegune Boğazköy Türgis) leping (CTH 51), ${ }^{1}$ mille sõlmisid Hetiidi kuningas Šuppiluliuma I (valitses u 1355-1320 eKr ${ }^{2}$ ) ja Mitanni ${ }^{3}$ kuningas Šattiwaza. ${ }^{4}$ See oluline leping ${ }^{5}$ on dateeritud 14. sajandisse $\mathrm{eKr}^{6}$ ja seda säilitatakse praegu Briti Muuseumis Londonis.

Käesolev leping oli vasallileping Hetiidi suurkuninga ja Mitanni valitseja Šattiwaza vahel millega Šuppiluliuma I tunnistas Šattiwazat kui tulevast Mitanni kuningat ja Šattiwaza tunnistas Šuppiluliumat kui oma isandat ehk siis sisuliselt sai temast Šuppiluliuma I vasall (Beckman 1999: 41-48).

\section{Lepingujumalaid Šuppiluliuma I ja Šattiwaza lepingus}

Uurijate tähelepanu pälvis ülal mainitud lepingu lõpuosas leiduv pikk jumalate nimekiri, milles esinevad muuhulgas järgmised jumalad:

[§17'] $\mathrm{A}_{1+2}$ Rs. 54', (226) DU EN ša-me-e ù er-se-ti ${ }^{\mathrm{D}} 30$ ù DUTU D30 URUKASKAL-na [A]N ù 'KI'-ti ${ }^{\mathrm{D} U} \mathrm{EN} k u$-ri-in-ni URU Ka-hat ${ }^{\mathrm{D}} \mathrm{GÌR} \check{s} a{ }^{\mathrm{URU}} \mathrm{Kùr} r$-dá Rs. 55', (227) U EN URU U-ḩu-šu-ma-an (227) É-a-LUGAL EN ha-sí-[s] iA-nu $A-{ }^{\circ} a n^{\prime}-t_{4}$ en.líl ù nin.líl DINGIR.MEš Mi-it-ra-aš-ši-il

Rs. 56', DINGIR.MEŠ Ú -ru-wa-na-aš-ši-il ${ }_{5}{ }^{\mathrm{D}} I n$-tar DINGIR.MEŠ Na-ša-at-ti-ia $-a n$ $n a(228)^{-\mathrm{D}} \mathrm{K}$ ASKAL.KUR $\check{a} a{ }^{\mathrm{D}} \check{S} a-m a-a[n]-m i-n u-h i{ }^{\mathrm{D} U}$ EN URU Wa-aššug-ga-an-ni

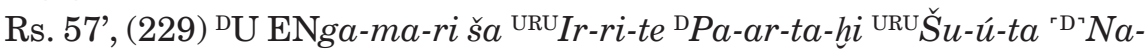

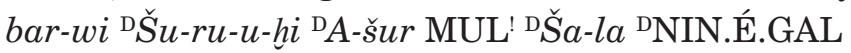

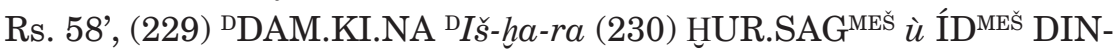
GIR $^{\text {MES̆ }}$ AN DINGIR ${ }^{\text {MES̆ }} \mathrm{KI}_{-} t i^{7}$

Rs. 54'-58' (226-230): Äikesejumal (piksejumal), taeva ja maa isand; kuujumal ning päikesejumal, Harrāni (linna) kuujumal (Su'en); taevas ja maa, äikese/piksejumal; Kahati kurinni isand; karjajumal (karjus-jumal), Ušumani isand; Ea-šarri, tarkuse isand; Anu, Antu, Enlil ja (jumalan-

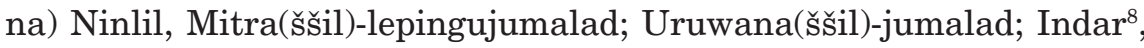
Našattijanna-jumalad, KASKAL.KUR ${ }^{9}$, Sammanminuhi, pikse(äikese) jumal, Waššukinni ${ }^{10}$ isand, pikse(äikese)jumal, kamari Irrite isand ${ }^{11}$, Šuda (linna) Paridage, Nabarbi, Šuruhe, (jumalanna) Ištar - Veenuse 
täht, Šala, Bēlet ekalli ${ }^{12}$, (jumalanna) Damkina ${ }^{13}$, Išharara ${ }^{14}$, mäed ja jõed (mägede ja jõgede jumalused), Taeva jumalad, allilma jumalad. ${ }^{15}$

Kõik nimetatud jumalad on kutsutud lepingu tunnistajateks, kes peavad tagama lepingust kinnipidamise (Thieme 1960: 315). Kui enamik lepingus nimetatud jumalusi on hetiidi ja Mesopotaamia (assüüria, babüloonia, hurri) või Süüria päritoluga, siis neli jumalust või jumalusterühma äratavad tähelepanu sellega, et nende nimed on indoeuroopa päritoluga. Kuna nende jumalate nimesid on transkribeeritud erinevalt, olgu siinkohal antud mitme uurija erinevad transkriptsioonid ja tõlked:

Kuzmina: Mitraššil, Uruwanaššil, Indara, Našatianna (Kuzmina 2007: 133);

Oldenberg: Mi-it-ra, U-ru-w-na, In-dar, Na-sa-at-ti-ia (Oldenberg 1983: $24)$;

Thieme: mi-it-ra, u-ru-ua-na, in-dar, na-ša-a(t-ti-ia) (Thieme 1960: 303);

Dumézil: mi-id-ra-aš-šil, u-ru-wa-na-as-ši-el (Dumézil 1988: 66);

Beckman: the Mitra-gods, the Varuna-gods, Indra, the Nasatya-gods (Beckman 1999: 47, 53)

Wilhelm: Mitraššil-jumalad, Uruwanaššil-jumalad, Indar, Našatijannajumalad (Wilhelm 2005: 120).

Uurijad juhivad tähelepanu asjaolule, et neile jumalanimedele leiduvad vasted veedades: Mitra-, Varuna-, Indra- ja Nasatya- (Bailey 1975: 2; Thieme 1960: 303; Puhvel 1996: 49; Vannuci 2011: 42; Nissen 2012: 111; Wilhlem 2005: 120, märkus 45). Viimast on seletatud tõsiasjaga, et Mitanni riiki valitsesid indoeuroopa päritoluga kuningad (Duchesne-Guillemin 1978: 186; Schmeja 1975: 5; Nissen 2012: 110-111). Sellele viitavad nii mõned Mitanni valitsejate nimed (näiteks Artatama, Tušratta) (Nissen 2012: 110-111; Kalda 1999: VI-VII), Mitanni tekstides leiduv hobusekasvatust puudutav terminoloogia (Thieme 1960: 301; Puhvel 1996: 49; Kalda 1999: VIII) kui ka mõiste mairya-, mida kasutati Mitanni sõjalise aristokraatia esindajate kohta (Widengren 1965: 23). Ajaloolased nimetavad Mitanni riigis valitsenud indoiraanlasi "paraindialasteks" või "protoaarjalasteks" (Thieme 1960: 301; Dumézil 2001: 107). Filoloogid nimetavad neid hõimudeks, kes kõnelesid indoaarja või protoaarja keeli (Puhvel 1996: 49).

Lepingu lõpus leiduv indoiraani jumalate loetelu on vanim teadaolev indoeuroopa jumalate loetelu. Stephan Schlensogi arvates, kes tugineb Georges Dumézili teooriale indoeurooplaste ühiskonna kolmikjaotusest ${ }^{16}$ ja nende "kolmetisest ideoloogiast" (idéologie tripartite), on lepingus nimetatud jumalad 
hästi kooskõlas indoeuroopa panteoni funktsionaalse kolmikjaotusega. Vastavalt sellele jagunevat indoeuroopa jumalad järgmistesse tüüpidesse - jumalad, kes esindavad maagilise ja juriidilise valitsemise funktsiooni; jumalad, kelle funktsiooniks on sõjaline jõud ning jumalad, kelle funktsiooniks on viljakuse ja majandusliku jõukuse edendamine. Vastavalt sellele esindavat Varuna ja Mitra valitsemise funktsiooni koos selle maagilise ja kohut mõistva aspektiga, Indra sõjalist jõudu ja Nasatjad toitvat funktsiooni (Schlensog 2006: 30). Samale teooriale tugineva Geo Widengreni arvates kehastab Varuna valitsemisfunktsiooni maagilist, Mitra aga juriidilist aspekti (Widengren 1954: 23). Viimase seisukohaga ei saa siiski nõustuda, sest Rigveeda järgi on maagilise ( $m a y a)$ valdajad mõlemad jumalad (RV III,61,7; V, 63,3, 4 ja 7). Küll võib aga olla nõus Arthur Christenseniga, kes arvab, et nimetatud jumalad ei esine lepingutekstis mitte seepärast, nagu oleksid nad seotud erinevate lepingus käsitletud teemadega (abielu jne), vaid seepärast, et nad oli Mitanni riiki valitsenud "paraindialaste” peajumalad (Dumézil 2001: 108).

Alates tänapäevaste Mitra-uuringute "isast" Franz Cumontist on peaaegu kõik Mitrast kirjutavad ning Šuppiluliuma ja Šattiwaza lepingut käsitlevad uurijad viidanud lepingus esinevale nimele $M i$-it-ra-aš-ši-il kui jumal Mitra kirjalikule esmamainimisele (Cumont 1975: 2-3; Vermaseren 1965: 9; Schwertheim 1979: 8; Merkelbach 1994: 4; Clauss 2012: 14; Turcan 1996: 196; Vollkommer 1992: 583; Gordon 2012: 966; Hensen 2013: 7; Vannucci 2011: 42-43; Witschel 2013: 201). Elmar Schwertheim arvab, et Mitra esinemine lepingutekstis ei ole juhuslik, vaid on kooskõlas tema nime tähendusega (vanaindia appellativ mitram tähendab neutraalses vormis "lepingut" või "sõprust", meessoo vormis mitrah "lepingupartnerit" või "sõpra" (Schwertheim 1979: 8). Ka Rainer Vollkommeri arvates seletab Mitra esinemist lepingutekstis tõsiasi, et Mitra oli liidujumal (Gott des Bundes) (Vollkommer 1992: 583). Jan Gonda meelest meenutab Mitra nime kõrvutiesinemine lepingutekstis veedades esinevat vormelt Mitra-Varuna (millele vastab Avestas Mitra-Ahura) (Gonda 1978: 82). Veelgi kaugemale läheb Georges Dumézil, kes väidab, et lepingutekstis esinevaid jumalate nimesid $M i$ -it-ra-aš-ši-il ja Ú-ru-wa-na-aš-ši-il tulekski lugeda Mitra-Varuna (Dumézil 2001: 21, 107-108).

Schwertheimi ja Vollkommeri väitega, et Mitra esinemine lepingus on seotud tema kui lepingujumala loomusega, ei saa nõustuda, sest kõik tekstis loetletud jumalad ja jumalannad on kutsutud lepingu tunnistajaks. Kummalisel kombel esineb aga lepingutekstis üks seik, millele uurijad tähelepanu ei juhi, nimelt vorm, milles Mitraks tõlgendatud jumalanimi esineb. Kui eelpool nimetatud uurijad, kes käsitlevad jumala nime esinemist lepingus jumal Mitra esmamainimisena kirjalikes allikates, käsitlevad nime ainsuslikuna (Mitra), 
siis õigesti annavad seda edasi oma tõlkes Gary Beckman ja Gernot Wilhelm, kes tõlgivad DINGIR.MEŠMi-it-ra-aš-ši-il "Mitra-jumalad" (Beckman) / "Mitraššiljumalad" (Wilhem). Paraku ei tee nimetatud tõlkijad sellisest nimevormist mingeid jäeldusi. Nii viitab Wilhelm joonealuses kommentaaris (nagu kõik eelpool nimetatud uurijadki) Mitrale ainsuses. Ometi viitab aga lepingutekstis esinev nimevorm selgelt mitmusele, st teatud jumalate grupile. Kuigi sel kujul on nimevorm singulaarne ja sellele ei leidu paralleele üheski teises muistses tekstis, pakuvad käesoleva artikli autorid välja hüpoteesi, millega saaks sellist nimekasutust seletada.

\section{Järeldused}

Mida antud juhul võib järeldada? Andmata siinkohal põhjalikumat ülevaadet jumal Mitra nime etümoloogiast ning selle tähendusnüanssidest ja tõlkevõimalustest, ${ }^{17}$ lähtuvad autorid sellest, et sõna mitra tähendab nii veedade kui ka avesta keeles "lepingut, liitu" ning teevad ettepaneku tõlkida akkadikeelset väljendit DINGIR.MEŠ $M i$-it-ra-aš-ši-il mitte pärisnimena ("Mitrad" või koos "jumalat" tähistava determinatiiviga "Mitra-jumalad"), vaid "lepingujumalad". Sel juhul ei oleks nimi lepingutekstis jumal Mitra esmamainimine, vaid viitaks anonüümsele jumalaterühmale, kelle ülesandeks oli lepingute/liitude kaitsmine. Kuigi lepingu tagajatena tuleb näha kõiki lepingu tekstis esinevaid jumalaid, väärib ometi tähelepanu, et nende hulgas mainitakse ka ühte sellele spetsialiseerunud gruppi. Viimaste nimi ja funktsioon seovad neid hilisemas india ja iraani usundis olulise jumal Mitra/Mithraga, kelles paljud uurijad on näinud mitte ainult kosmilise- ja sotsiaalse korra ning lepingute ja kokkulepete kaitsjat, vaid lausa lepingu jumalikku personifikatsiooni (Meillet 1907: 143-159; Thieme 1957; Thieme 2015: 1998-2009; Gershevitsch 1967: 26; Gonda 1972: 102 jj; Puhvel 1978: 335-455; Bonfante 1978: 57; Turcan 1996: 196).

\section{Lühendid}

CTH = Laroche, E., Catalogue des textes hittites, Paris: Klincksieck 1971.

$\mathrm{KBo}=$ Keilschrifttexte aus Boghazköi.

KUB = Keilschrifturkunden aus Boghazköy.

RV - Rigveeda 


\section{Kommentaarid}

* Artikli ilmumist toetab Tartu Ülikooli baasfinantseeritav projekt PHVAJ 17908.

1 Vt teksti Jankowski \& Wilhelm 2005: 113-121. Lepingu ajaloolise tausta kohta vt samas: 113-114; Thieme 1960: 301-317; Mayrhofer 1974; Wilhelm 1982. Leping Šuppiluliuma ja Šattiwaza vahel (CTH.51) oli kirjutatud nii akkadi kui hetiidi keeles. Akkadikeelne versioon säilis paremini (Beckman 1999: 41). Akkadikeelne dokument kiilkirjas: KBo 1.1, KBo 1.2, KUB 3.1 a-d+KBo 28.111+114 (+) 113; Hetiidikeelne dokument kiilkirjas: KUB 21.18 (+) KUB 26.34.

2 Šuppiluliuma I valitsemisaega on võimalik dateerida erinevalt (nt 1380-1340 eKr või 1355-1320 eKr jne), sest tänapäeva Vana-Lähis-Ida uuringutes on kasutusel kuni neli erinevat kronoloogiasüsteemi Vana-Lähis-Ida ajaloo kohta: pikk, keskmine, lühike ja ülilühike kronoloogia (Sazonov \& Espak \& Johandi 2017: 42-43). Siinkirjutajad kasutavad lühikest kronoloogiat. Vt Niemeier 2008: 315.

${ }^{3}$ Mitanni ehk Hanigalbat oli hurriidi riik Põhja-Mesopotaamias ca 1500-1300 eKr.

4 Varem on tema nime loetud ka kui Matiwaza või Kurtiwaza (Duchesne-Guillemin 1978: 187).

5 Hetiitide lepingutest vt Devecchi 2012: 637-645.

6 Teksti täpsemad dateeringud on siiski kõikuvad: 14. saj I pool (Witschel 2013: 201); u 1400 eKr (Oldenberg 1983: 24); 1360 eKr (Schmeja 1975: 5); u 1375-50 eKr (Bailey 1975: 2); 1380 eKr (Gonda 1978: 82); u 1380 eKr (Dumézil 2001: 107; Turcan 1996: 196; Turcan 1981: 7; Vollkommer 1992: 583; Puhvel 1996: 49; Schlensog 2006: 30); kõikumiste põhjuseks on märkuses 2 nimetatud kronoloogilised probleemid.

7 Transkr. von G. Wilhelm, "Der Vertrag Šuppiluliumas I. von Hatti mit Šattiwazza von Mittani" (CTH 51.I), http://www.hethport.uni-wuerzburg.de/txhet_svh/exemplar. php?xst=CTH\%2051.I\&expl=A\&lg=DE\&ed=G.\%20Wilhelm; G. Beckman, http://www. heritageinstitute.com/zoroastrianism/ranghaya/suppiluliuma_shattiwaza_treaty.htm (viimane külastus 25.01.2019); Wilhelm 2005: 113-121; vt ka G. Beckmani tõlget (Beckman 1999: uj42-54).

8 Ilmselt jumal Indra.

9 Antud jumalus pole tuvastatav. KASKAL sumeri keeles "tee", "teekond", "karavan", KUR - maa, tuul, allilm. Tõenäoliselt mingi allilma jumalus (allilma vee vool) (Beckman 1999: 47).

${ }^{10}$ Mitanni riigi pealinn.

${ }^{11}$ Beckman pakub (1999: 47): "Irrite templi platvormi".

12 Jumalanna, nimi tähendab "palee emand".

13 Jumalanna, jumal Enki abikaasa. Variant: Damgalnuna.

14 Tundmatut päritolu jumalanna, algselt pärit Süüriast. Armastusejumalanna. Sageli samastatud Ištariga.

${ }^{15}$ Vladimir Sazonovi tõlge - katkend Hetiidi kuninga Šuppiluliuma I ja Mitanni kuninga Šattiwaza lepingust. 
${ }^{16}$ Selle teooria järgi eristasid muistsed indoeuroopa hõimud ühiskonnas kolme funktsiooni - preesterlik-maagilist, sõjalist ja produktiivset funktsiooni ning vastavalt sellele jagunes nende ühiskond kolmeks seisuseks - preestriteks, valitsejateks-sõjameesteks ja maaharijateks-käsitöölisteks.

17 Selle kohta vt Puhvel 1978: 335-355; Schmidt 1978: 345-394; Bonfante 1978: 47-57; Thieme 1975: 21-33; Lahe \& Kalda 2016: 133-135.

\section{Kirjandus}

Bailey, Harold W. 1975. The second stratum of the Indo-Iranian gods. Hinnells, John R. (toim). Mithraic Studies. Proceedings of the First International Congress of Mithraic Studies 1. Manchester: Manchester University Press, lk 1-20.

Beckman, Gary 1999. Hittite Diplomatic Texts. Second Edition. Writings from the Ancient World Series. Atlanta, Georgia: Society of Biblical Literature Scholars Press.

Bonfante, Giulio 1978. The Name of Mithra. Études Mithraiques. Acta Iranica 17, lk 47-57.

Clauss, Manfred 2012. Mithras. Kult und Mysterium. Darmstadt: Wissenschaftliche Buchgesellschaft.

Cumont, Franz 1975. Die Mysterien des Mithra. Ein Beitrag zur Religionsgeschichte der römischen Kaiserzeit. Autorisierte Deutsche Ausgabe von Georg Gehrich. Fünfte Auflage. Unveränderter Nachdruck der von Kurt Latte besorgten dritten, vermehrten und durchgesehene Auflage von 1923. Darmstadt: Wissenschaftliche Buchgesellschaft.

Devecchi, Elena 2012. "Treaties and Edicts in the Hittite World". Organization, Representation, and Symbols of Power in the Ancient Near East. Proceedings of the 54th Rencontre Assyriologique Internationale at Würzburg 20-25 July 2008. Gernot Wilhelm (toim). Winona Lake, Indiana, Eisenbrauns, lk 637-645.

Duchesne-Guillemin, Jaques 1978. Iran and Greece in Commagene. Duchesne-Guillemin, Jaques (toim). Études Mithriaques. Actes du 2e congrès international, Téhéran 1-8 sept. 1975. Acta Iranica, série I. Leiden: Diff. E. J. Brill, lk 187-200.

Dumézil, Georges 1988. Mitra-Varuna. An Essay on Two Indo-European Representations of Sovereignty. New York: Zone.

Dumézil, Georges 2001. Indoeurooplaste mü̈̈did ja jumalad. Tlk Marri Amon \& Lembe Lokk. Tallinn: Varrak.

Gershevitch, Ilya 1967. The Avestan Hymn to Mithra. With an introduction, translation and commentary by Ilya Gershevitch. Cambridge: Cambidge University Press.

Gonda, Jan 1972. The Vedic god Mitra. Leiden: E. J. Brill.

Gonda, Jan 1978. Die Religionen Indiens. I Veda und älterer Hinduismus (Die Religionen der Menschheit 11). 2., überarbeitete und erg. Aufl. Stuttgart et al.: Kohlhammer. 
Gordon, Richard 2012. Mithras (Mithraskult). Reallexikon für Antike und Christentum. Bd. XXIV. Stuttgart: Anton Hiersemann, lk 964-1009.

Hensen, Andreas 2013. Mithras. Der Mysterienkult an Limes, Rhein und Donau. Limesmuseum Aalen. Zweigmuseum des Arhäologischen Landesmuseum Baden-Württemberg. Darmstadt: Wissenschaftliche Buchgesellschaft.

Jankowski, Bernd \& Wilhelm, Gernot (toim) 2005. Staatsverträge, Herrscherinschriften und andere Dokumente zur politischen Geschichte (Texte aus der Umwelt des Alten Testaments, Neue Folge, Bd. 2). Gütersloh: Gütersloher Verlagshaus.

Kalda, Martti 1999. RgVeeda mütoloogia. I-II. Tallinn (käsikiri).

Kuz'mina, Elena E. 2007. The Origin of the Indo-Iranians. Leiden, Boston: Brill.

Lahe, Jaan \& Kalda, Martti 2016. Mitra kuju Indias ja Mithra kuju Iraani usundites uurimus võrdlevast mütoloogiast. Mäetagused 65, lk 117-160 (doi: 10.7592/MT2016.65. lahe_kalda).

Mayrhofer, Manfred 1974. Die Arier im Vorderen Orient - ein Mythos? Wien: VÖAW.

Meillet, Paul Jules Antoine 1907. Le dieu Indo-Iranien Mitra. Journal asaiatique 10, lk 143-159.

Merkelbach, Reinhold 1994. Mithras. Ein persisch-römischer Mysterienkult. 2. Aufl. Weinheim: Beltz Athenäum.

Niemeier, Wolf-Dietrich 2008. Hattusas Beziehungen zu West-Kleinasien und dem mykenischen Griechenland (Ahhiyawa). Wilhelm, Gernot (toim). Hattuša-Boğazköy. Das Hethiterreich im Spannungsfeld des Alten Orients (Colloquien der Deutschen OrientGesellschaft, Bd. 6) Wiesbaden: Harrassowitz (https://vdocuments.mx/niemeier-hattusasbeziehungen-zu-west-kleinasien.html - 29. jaanuar 2019).

Nissen, Hans J. 2012. Geschichte Alt-Vorderasiens (Oldenbourg Grundriss der Geschichte, Bd. 25). München: Oldenbourg.

Oldenberg, Hermann 1983. Die Religion des Veda. Stuttgart: Magnus-Verlag.

Puhvel, Jaan 1978. Mithra as an Indo-European Divinity. Études Mithraiques. Acta Iranica 17 , lk 335-355.

Puhvel, Jaan 1996. Võrdlev mütoloogia. Eesti mõttelugu 12. Tartu: Ilmamaa.

Sazonov, Vladimir \& Espak, Peeter \& Johandi, Andreas 2017. Vana-Lähis-Ida ajalugu 3500-2000 eKr. Mesopotaamia ajalugu kiilkirja tekkest kuni Uus-Sumeri languseni. Tartu: Tartu Ülikooli kirjastus (https://www.researchgate.net/profile/ Peeter_Espak/publication/298045464_Vana-Lahis-Ida_ajalugu_3500-2000_eKr/ links/56e55bc708ae68afa1128f51/Vana-Laehis-Ida-ajalugu-3500-2000-eKr.pdf 29. jaanuar 2019).

Schlensog, Stephan 2006. Der Hinduismus. Glaube, Geschichte, Ethos. München, Zürich: Piper.

Schmeja, Hans 1975. Iranisches und Griechisches in den Mithrasmysterien (Innsbrucker Beiträge zur Sprachwissenschaft. Vorträge 13). Innsbruck: H. Kowatsch. 
Schmidt, Hans-Peter 1978. Indo-Iranian Mitra Studies: The State of the Central Problem. Études Mithraiques. Acta Iranica 17, lk 345-394.

Schwertheim, Elmar 1979. Mithras. Seine Denkmäler und sein Kult. Antike Welt 10. Sondernummer.

Thieme, Paul 1957. Mitra and Aryaman. New Haven, CT: Yale University Press.

Thieme, Paul 1960. The "Aryan" gods of the Mitanni treaties. Journal of American Oriental Society 80 (4), lk 301-317.

Thieme, Paul 1975. The concept of Mitra in Aryan belief. Hinnells, John R. (toim). Mithraic Studies. Proceedings of the First International Congress of Mithraic Studies I. Manchester: Manchester University Press, Rowman and Littlefield, lk 21-33.

Thieme, Paul 2015. Mithra Avestas. Akadeemia 11, lk 1998-2009 (https://www.digar. ee/viewer/et/nlib-digar:261497/187941/page/78 - 30. jaanuar 2019).

Turcan, Robert 1981. Mithra et le mithriacisme. Paris: Presses Universitaires de France. Turcan, Robert 1996. The Cults of the Roman Empire. Oxford: Blackwell.

Vannucci, Marta 2011. The Vedic God Mitra. A Concise Study. New Delhi: D. K. Printworld (P) Ltd. Publishers of Indian traditions.

Vermaseren, Maarten J. 1965. Mithras. Geschichte eines Kultes. Stuttgart: W. Kohlhammer.

Vollkommer, Rainer 1992. Mithras. Lexicon Iconographicum Mythologiae Classicae. VI.1. Zürich \& München: Artemis Verlag, lk 583-626.

Widengren, Geo 1954. Stand und Aufgaben der iranischen Religionsgeschichte. Numen 1, lk 17-83 (doi: 10.1163/156852755X00033).

Widengren, Geo 1965. Die Religionen Irans. Die Religionen der Menschheit 14. Stuttgart: Kohlhammer.

Wilhelm, Gernot 1982. Grundzüge der Geschichte und Kultur der Hurriter. Darmstadt: Wissenschaftliche Buchgesellschaft.

Wilhelm, Gernot 2005. Der Vertrag Šuppiluliumas I. von Hatti mit Šattiwazza von Mitanni. Staatsverträge, Herrscherinschriften und andere Dokumente zur politischen Geschichte. Texte aus der Umwelt des Alten Testaments. Neue Folge 2, lk 113-121.

Witschel, Christian 2013. Die Ursprünge des Mithras-Kults. Orientalischer Gott oder westliche Neuschöpfung? Imperium der Götter. Isis - Mithras - Christus: Kulte und Religionen im Römischen Reich. Stuttgart: Konrad Theiss Verlag, lk 200-210. 


\section{Summary}

\section{First mention of Mitra in the treaty between the Hittite king Šuppiluliuma I and the Mittannian ruler Šattiwaza?}

\section{A short study into the Indo-Iranian religion}

\section{Jaan Lahe}

Professor of Biblical Studies, Institute of Theology of the Estonian Evangelical Lutheran Church

Associate Professor of Cultural and Religious Studies, Tallinn University, School of Humanities

usundiuurija@gmail.com

\section{Vladimir Sazonov}

Research Fellow, Estonian Academy of Security Sciences

Senior Research Fellow in Ancient Near Eastern Studies, University of Tartu

vladimir.sazonov@ut.ee

Keywords: gods of treaties, Hittites, Indo-Europeans, Mitra, state of Mitanni, Šattiwaza, treaty, Šuppiluliuma I

In 1907 a treaty was found in the Hittite royal archive in Boğazköy. It was forged between the Hittite king Šuppiluliuma I and the Mitanni king Šatiwaza. Among the deities named in this contract is DINGIR.MEŠ Mi-it-ra-aš-ši-il. This is usually understood to refer to the god Mitra. However, the authors of this article draw attention to the fact that the name occurs in the plural form; the divine classifier DINGIR ("god" before Mitra's name) is written in the plural form DINGIR.MEŠ, meaning "gods". They are of the opinion that it should not be understood as a proper name but as a denomination of a group of deities that one could translate as "gods of the treaties". 